\title{
Effect of optimal multi-DG siting and sizing in transmission system using hybrid optimization technique for voltage control
}

\author{
Sharifah A. Shaaya ${ }^{1}$, Ismail Musirin ${ }^{2}$, Shahril I. Sulaiman ${ }^{3}$, Mohd H. Mansor ${ }^{4}$, \\ Sharifah A. S. Mustaffa ${ }^{5}$ \\ ${ }^{1,4,5}$ Department of Electrical \& Electronics Engineering, Universiti Tenaga Nasional, Malaysia \\ ${ }^{2,3}$ Faculty of Electrical Engineering, Universiti Teknologi MARA, Malaysia
}

\begin{tabular}{l} 
Article Info \\
\hline Article history: \\
Received Jun 10, 2019 \\
Revised Aug 12, 2019 \\
Accepted Aug 26, 2019 \\
\hline
\end{tabular}

Keywords:

Distributed generation, Hybrid optimization, Immunized-brainstormevolutionary programming Voltage control Multi-DG

\begin{abstract}
The advancement of Distributed Generation (DG) technologies have caused great impact to power system operation. Inappropriate installation of DGs may lead to over-compensation or under-compensation situation. Thus, a reliable optimization is urgent to avoid any unwanted effect. This paper analyses the installation impact of different types-multi-DGs determined using a pre-developed hybrid optimization technique termed as ImmunizedBrainstorm-Evolutionary Programming (IBSEP). It is imperative to study the effect of multi-DGs installation such that a relevant utility can make a correct decision, whether its installation is worth or vice versa. Rigorous study has been conducted in terms of identifying the optimal location and sizing, installed on transmission system for voltage control involving different DG types. Comprehensive results are embedded in this paper to demonstrate the effect of multi-DGs installation in transmission system which in turns beneficial to the utility.
\end{abstract}

Copyright $(2020$ Institute of Advanced Engineering and Science. All rights reserved.

Corresponding Author:

Sharifah A. Shaaya,

Department of Electrical Engineering,

Universiti Tenaga Nasional,

Jalan Ikram-Uniten, 43000, Kajang, Selangor, Malaysia.

Email:shazwa@uniten.edu.my

\section{INTRODUCTION}

Voltage profile of a power system is one of important parameters to be controlled by power system provider. While power system losses could be translated into monetary loss to the power provider, uncontrolled voltage profile could cause imbalance network and threaten the network safety. Voltage level is easily affected by changes in reactive load. Failing to retain the voltage level to a permissible limit may results in voltage collapse. Ways to avoid this adverse effect had been studied by other researchers that involve installing static VAR compensator in power network, controlling the voltage and VAR output of generators and denial of demand during critical under-voltage event [1].

Distributed generation (DG) is generally defined as small-scale electricity generation. Due to its size, a DG is usually located at the distribution side of power system, to be as close as possible to the consumer side, although DG placement at the transmission side is also permissible [2, 3]. Assessments on DG penetration to power network were continuously done by many researchers that conclude carefully selected DG size and location may enhance the voltage profile and reduce power loss of that network [4-7]. Commonly done at the distribution side, studies on the effects of optimally selected DG installation were conducted at the transmission network as well [8-12]. These works concluded that medium sized DGs, in particular the pure real power delivering DGs, were able to reduce transmission network loss with improved voltage profile. Few studies were conducted to assess effect of installing other DGs that have the 
ability to deliver or absorb reactive power $[13,14]$. These studies found that different type of DG will affect power network performance differently.

Inventing robust and reliable optimization technique to determine optimal DG location and size is currently trending in the researchers community, aiming at alleviating setbacks in the existing optimization techniques [15-20]. Apart from finding higher probability of optimum solution towards meeting the objective functions, new optimization algorithms are developed to reduce computational burden in the classical optimization techniques $[21,22]$

Brainstorm Optimization (BSO) is a newly developed algorithm that made its appearance in 2011. An algorithm that mimics the collective behavior of human has caught attention of many researcherst. Though it is proven reliable in solving science and engineering problems, it does has high computational burden due to its K-means clustering technique as well as easily trapped in the local maxima [23-26]. In this paper, an optimization technique that embed Artificial Immune System (AIS) optimization technique and BSO into the frame of Evolutionary Programming (EP) algorithm is used to determine optimal locations and sizes of multiple DGs, of different power delivering capabilities, in power transmission system for voltage control. The hybrid technique is termed as Immunized-Brainstorm-Evolutionary Programming (IBSEP).

\section{RESEARCH METHOD}

Main objective of this study is to compare the capabilities of different types of DGs in improving transmission system's minimum voltage profile. DGs are categorized into four major types, based on their ability to deliver power. In this paper, comparison between the performance of these four DG types to control system voltage will be investigated, based on the definition below:

Type-1. This type of DGs deliver only real power. Examples of Type-1 DG are photovoltaic, microturbines and fuel cells. In this paper, Type-1 DGs will be referred to as T1 from this point onwards.

Type-2. DGs that fall under this category are the ones able to deliver only reactive power. Example of this DG type is any DGs based on synchronous compensator. Type-2 DGs will be referred to as T2 from this point onwards.

Type-3. This type of DG is able to deliver both real and reactive power. Cogeneration and gas turbine are example of Type-3 DG. Type-3 DGs will be referred to as T3 from this point onwards.

Type-4. DGs under this category are capable of delivering real power, but consume reactive power. Example of Type-4 DGs are doubly fed induction generator (DFIG) and induction generators used in wind farms. Type-4 DGs will be referred to as T4 from this point onwards.

In this assessment, multi-DG units set to be 2, 3 and 5 units for each DG type are to be inserted to the network. For T1 DG, the real power is limited to 50MW, based on the maximum PV output tabulated in [27]. In terms of apparent power S, each T1 DG will be limited to 50MVA, based on (1). For fair comparison, the power of other DG types are then limited to 50MVA each.

$$
S=\sqrt{P^{2}+Q^{2}}
$$

Where $S$ is the apparent power, $P$ is the real power and $Q$ is the reactive power of the DG.

Transmission system minimum voltage profile before DGs' placement in the system will be compared with the system voltage profile after multiple DGs placement, for each DG type. The objective function to be met is to improve system's minimum voltage profile, represented mathematically as (2),

$$
O \cdot F=V_{(\min , o p t)}>V_{(\text {min,pre-opt })}
$$

The objective function is however subjected to power balance equality constraint as (3)

$$
\sum_{i=1}^{n} P_{i}=P_{\text {demand }}+P_{\text {loss }}
$$

as well as inequality constraints:

$$
\begin{aligned}
& P_{i, \min } \leq P_{i} \leq P_{i, \max } \\
& V_{\min } \leq V_{i} \leq V_{\max }
\end{aligned}
$$

where $P_{\text {demand }}$ is total system load demand, $P_{\text {loss }}$ is the total system loss, and $P_{i, \min }$ and $P_{i, \max }$ are minimum and maximum real power output of $i^{\text {th }}$ generator, respectively. Following IEEE standard, $V_{\min }$ and $V_{\max }$ should be 0.95 p.u. and 1.05 p.u. respectively. 


\subsection{Proposed Methodology}

In this work, DGs are placed into IEEE-30 RTS to compensate system loss while load increased. In order to maximize the voltage profile enhancement, optimal sized DGs must be injected at optimal location. These optimal sized DG and optimal bus location were determined using IBSEP optimization technique. IBSEP technique is explained below.

\subsubsection{Proposed Immunized-Brainstorm-Evolutionary Programming (IBSEP)}

Flowchart for optimal size and location of DG to improve voltage profile using IBSEP is shown in Figure 1. The process is briefly explained afterwards:

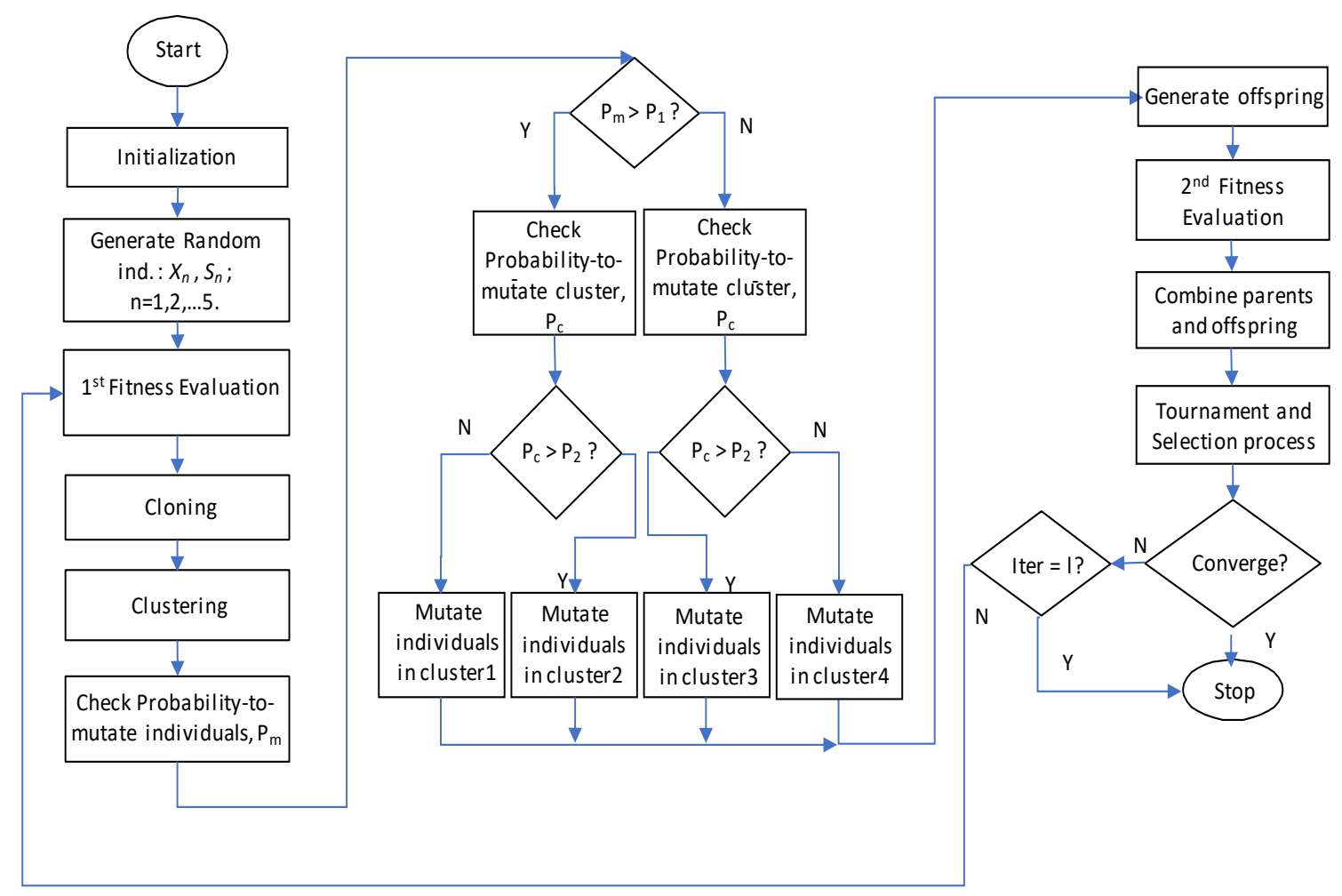

Figure 1. Flowchart for optimal DG location and sizes using IBSEP technique

Step 1: Initialization of population number $n$, number of clusters $l$, probability-to-mutate cluster, $p_{1}$ and $p_{2}$, reactive load, $Q_{d}$ and location of where $Q_{d}$ will be incremented. Random DG location, $X_{n}$ and size, $S_{n}$ are generated. $n$ represents DG's unit number.

Step 2: Fitness of the transmission system, i.e. the minimum system's voltage profile with optimal DG installation will be determined from load flow. Only individuals capable of increasing the minimum voltage profile are selected. These individuals, or parents, are then cloned to increase the population. The grown population is then divided into few clusters.

Step 3: Random number $p_{m}$ and $p_{c}$ are then generated. They are used to decide which cluster to be mutated. Gaussian mutation operator is used to generate new individuals, known as offspring. Load flow is then run to determine the fitness of the offspring.

Step 4: The offspring and the parents are then combined to compete in a tournament process. Only $n$ numbers of individuals with best fitness will be transcribed to the next process.

Step 5: A convergence test will then take place. In this test, the difference between the highest and lowest fitness values will calculated. Mathematically, it is written as

$$
\text { Voltage }_{\max }-\text { Voltage }_{\min } \leq 0.00001
$$

Should this condition is met, optimal DG locations and sizes will be recorded. Otherwise, Step 2 until Step 5 are to be repeated. 


\section{RESULTS AND ANALYSIS}

Reactive load, Qd, on one of load buses of IEEE-30 RTS was incremented, from 0 MVar to 30 MVar. Optimal DGs are to be installed at few load buses to compensate the losses due to the increased demand. Two load buses were chosen for the added reactive loading; Bus-6 and Bus-30. These buses represent the healthy and the weak buses of IEEE-30 RTS respectively, identified from a pre-loadability test. While the reactive loading was increased, the unit of DGs was varied; 2, 3, and 5 units, for each DG types. The effect of different DG types and amount on system's voltage profile and losses are presented below.

\subsection{Effect of Optimal DG Placement on System Voltage Profile While Reactive Load Varies at Bus-6}

Table 1 tabulates the system's voltage profile and system's loss without DGs insertion. Table 2 lists system's voltage profiles with optimal DGs for each DG types and the percentage of voltage profile enhancement percentage (VEP), calculated using (7).

$$
V E P=\frac{\text { Volt }_{D G}-\text { Volt }_{\text {pre }- \text { opt }}}{\text { Volt }_{\text {pre }- \text { opt }}} \times 100 \%
$$

Table 1. System Loss and Voltage Profile of IEEE-30 RTS without Optimal DG when Reactive Load $\mathrm{Q}_{\mathrm{d}}$

\begin{tabular}{cccccc}
\multicolumn{5}{c}{ Increases at Bus-6 and Bus-30 } \\
\hline $\begin{array}{c}\mathrm{Q}_{\mathrm{d} 6} \\
\text { (Mvar) }\end{array}$ & $\begin{array}{c}\text { Pre-opt Loss } \\
\text { (MW) }\end{array}$ & $\begin{array}{c}\text { Pre-opt Voltage } \\
\text { (p.u) }\end{array}$ & $\begin{array}{c}\mathrm{Q}_{\mathrm{d} 30} \\
(\text { Mvar) }\end{array}$ & $\begin{array}{c}\text { Pre-opt Loss } \\
\text { (MW) }\end{array}$ & $\begin{array}{c}\text { Pre-opt Voltage } \\
\text { (p.u) }\end{array}$ \\
\hline 0 & 17.60 & 0.9945 & 0 & 17.56 & 1.0036 \\
10 & 17.71 & 0.9907 & 10 & 18.11 & 0.9326 \\
20 & 17.76 & 0.9892 & 20 & 19.55 & 0.8438 \\
30 & 17.82 & 0.9877 & 30 & 23.44 & 0.7069 \\
\hline
\end{tabular}

Table 2 shows that IBSEP technique was able to calculate optimal location and optimal size of every DG types in order to enhance the system's minimum voltage profile when reactive load was increased at Bus-6. T2 and T3 DGs show good consistency in enhancing the minimum voltage profile the most, as indicated by highest VEP values in bold. Except for T4 DGs, other optimal DG types were able to improve the minimum voltage profile, if not the same, when DG quantity was added.

Table 2. IEEE-30 RTS Voltage Profile and Voltage Enhancement Percentage (VEP) with Optimal DGs While $\mathrm{Q}_{\mathrm{d}_{6}}$ Increases

\begin{tabular}{cccccccccc}
\hline $\begin{array}{c}\mathrm{Q}_{\mathrm{d} 30} \\
\text { MVar) }\end{array}$ & $\begin{array}{c}\text { DG Amt. } \\
\text { (Unit) }\end{array}$ & $\begin{array}{c}\mathrm{VP}_{\mathrm{T} 1} \\
(\mathrm{p} . \mathrm{u})\end{array}$ & $\begin{array}{c}\mathrm{VP}_{\mathrm{T} 2} \\
(\mathrm{p} . \mathrm{u})\end{array}$ & $\begin{array}{c}\mathrm{VP}_{\mathrm{T} 3} \\
(\mathrm{p} . \mathrm{u})\end{array}$ & $\begin{array}{c}\mathrm{VP}_{\mathrm{T} 4} \\
(\mathrm{p} . \mathrm{u})\end{array}$ & $\begin{array}{c}\mathrm{VEP}_{\mathrm{T} 1} \\
(\%)\end{array}$ & $\begin{array}{c}\mathrm{VEP}_{\mathrm{T} 2} \\
(\%)\end{array}$ & $\begin{array}{c}\mathrm{VEP}_{\mathrm{T} 3} \\
(\%)\end{array}$ & $\begin{array}{c}\mathrm{VEP}_{\mathrm{T} 4} \\
(\%)\end{array}$ \\
\hline \multirow{2}{*}{0} & 2 & 1.0076 & 1.0100 & 1.0100 & 1.0000 & 1.31 & $\mathbf{1 . 5 6}$ & $\mathbf{1 . 5 6}$ & 0.55 \\
& 3 & 1.0084 & 1.0100 & 1.0100 & 1.0200 & 1.40 & 1.56 & 1.56 & $\mathbf{2 . 5 6}$ \\
& 5 & 1.0100 & 1.0100 & 1.0100 & 1.0100 & $\mathbf{1 . 5 6}$ & $\mathbf{1 . 5 6}$ & $\mathbf{1 . 5 6}$ & $\mathbf{1 . 5 6}$ \\
10 & 2 & 1.0064 & 1.0100 & 1.0100 & 0.9926 & 1.59 & $\mathbf{1 . 9 5}$ & $\mathbf{1 . 9 5}$ & 0.19 \\
& 3 & 1.0072 & 1.0100 & 1.0100 & 0.9918 & 1.67 & $\mathbf{1 . 9 5}$ & $\mathbf{1 . 9 5}$ & 0.11 \\
& 5 & 1.0093 & 1.0100 & 1.0100 & 1.0000 & 1.88 & $\mathbf{1 . 9 5}$ & $\mathbf{1 . 9 5}$ & 0.94 \\
20 & 2 & 1.0052 & 1.0100 & 1.0100 & 0.9900 & 1.62 & $\mathbf{2 . 1 1}$ & $\mathbf{2 . 1 1}$ & 0.08 \\
& 3 & 1.0060 & 1.0100 & 1.0100 & 1.0000 & 1.71 & $\mathbf{2 . 1 1}$ & $\mathbf{2 . 1 1}$ & 1.09 \\
& 5 & 1.0081 & 1.0100 & 1.0100 & 1.0000 & 2.07 & $\mathbf{2 . 1 1}$ & $\mathbf{2 . 1 1}$ & 1.09 \\
& 2 & 1.0040 & 1.0100 & 1.0100 & 1.0000 & 1.50 & $\mathbf{2 . 1 1}$ & $\mathbf{2 . 1 1}$ & 1.09 \\
& 3 & 1.0048 & 1.0100 & 1.0100 & 1.0000 & 1.58 & $\mathbf{2 . 1 1}$ & $\mathbf{2 . 1 1}$ & 1.09 \\
& 5 & 1.0070 & 1.0100 & 1.0100 & 1.0000 & 1.80 & $\mathbf{2 . 1 1}$ & $\mathbf{2 . 1 1}$ & 1.09 \\
\hline
\end{tabular}

\subsection{Effect of Optimal DG Placement on System Voltage Profile While Reactive Load Varies at Bus-30}

Increasing Qd30 at Bus-30 had caused the network to be in not-healthy mode, as indicated in Table 1. Table 3 tabulates the effect of different optimal DG types and amount on system' minimum voltage profile. Bold fonts indicate highest VEP value. When there is no load at Bus-30, all optimal DG types enhanced the minimum voltage profile, except when optimal T4 DGs were less than five units. As Qd30 increases, all optimal DGs arrangements were able to increase the system's minimum voltage profile where highest VEP was either due to T2 or T3 DGs. IBSEP technique was able to determine optimal T2 and T3 that consistently increase the voltage profile as amount of DG increases. However, increased minimum voltage profile does not mean that the network is in the healthy mode, shown by VP values for T1 DGs in italics. 
Table 3. IEEE-30 RTS Voltage Profile and Voltage Enhancement Percentage (VEP) with Optimal DGs While $\mathrm{Q}_{\mathrm{d}_{30}}$ Increases

\begin{tabular}{cccccccccc}
\hline $\begin{array}{c}\mathrm{Q}_{\mathrm{d} 30} \\
(\mathrm{MVar})\end{array}$ & $\begin{array}{c}\mathrm{DG} \text { Amt. } \\
\text { (unit) }\end{array}$ & $\begin{array}{c}\mathrm{VP}_{\mathrm{T} 1} \\
(\mathrm{p} . \mathrm{u})\end{array}$ & $\begin{array}{c}\mathrm{VP}_{\mathrm{T} 2} \\
(\mathrm{p} . \mathrm{u})\end{array}$ & $\begin{array}{c}\mathrm{VP}_{\mathrm{T} 3} \\
(\mathrm{p} . \mathrm{u})\end{array}$ & $\begin{array}{c}\mathrm{VP}_{\mathrm{T} 4} \\
(\mathrm{p} . \mathrm{u})\end{array}$ & $\begin{array}{c}\mathrm{VEP}_{\mathrm{T} 1} \\
(\%)\end{array}$ & $\begin{array}{c}\mathrm{VEP}_{\mathrm{T} 2} \\
(\%)\end{array}$ & $\begin{array}{c}\mathrm{VEP}_{\mathrm{T} 3} \\
(\%)\end{array}$ & $\begin{array}{c}\mathrm{VEP}_{\mathrm{T} 4} \\
(\%)\end{array}$ \\
\hline \multirow{2}{*}{0} & 2 & 1.0100 & 1.0100 & 1.0100 & 1.0036 & $\mathbf{0 . 6 4}$ & $\mathbf{0 . 6 4}$ & $\mathbf{0 . 6 4}$ & 0.00 \\
& 3 & 1.0100 & 1.0100 & 1.0100 & 1.0036 & $\mathbf{0 . 6 4}$ & $\mathbf{0 . 6 4}$ & $\mathbf{0 . 6 4}$ & 0.00 \\
& 5 & 1.0100 & 1.0100 & 1.0100 & 1.0300 & 0.64 & 0.64 & 0.64 & $\mathbf{2 . 6 3}$ \\
\multirow{2}{*}{10} & 2 & 0.9949 & 1.0083 & 1.0096 & 1.0000 & 6.68 & 8.11 & $\mathbf{8 . 2 6}$ & 7.22 \\
& 3 & 1.0002 & 1.0100 & 1.0100 & 1.0000 & 7.24 & $\mathbf{8 . 3 0}$ & $\mathbf{8 . 3 0}$ & 7.22 \\
& 5 & 1.0091 & 1.0100 & 1.0100 & 0.9900 & 8.20 & $\mathbf{8 . 3 0}$ & $\mathbf{8 . 3 0}$ & 6.15 \\
20 & 2 & 0.9241 & 1.0076 & 1.0083 & 1.0000 & 9.53 & 19.42 & $\mathbf{1 9 . 5 0}$ & 18.52 \\
& 3 & 0.9492 & 1.0100 & 1.0100 & 0.9900 & 12.50 & $\mathbf{1 9 . 7 0}$ & $\mathbf{1 9 . 7 0}$ & 17.33 \\
& 5 & 0.9488 & 1.0100 & 1.0100 & 0.9900 & 12.45 & $\mathbf{1 9 . 7 0}$ & $\mathbf{1 9 . 7 0}$ & 17.33 \\
& 2 & 0.8199 & 1.0068 & 0.9994 & 1.0000 & 15.99 & $\mathbf{4 2 . 4 3}$ & 41.39 & 41.47 \\
& 3 & 0.8430 & 1.0096 & 1.0100 & 1.0000 & 19.26 & 42.83 & $\mathbf{4 2 . 8 9}$ & 41.47 \\
& 5 & 1.0000 & 1.0100 & 1.0100 & 0.9900 & 41.47 & $\mathbf{4 2 . 8 9}$ & $\mathbf{4 2 . 8 9}$ & 40.06 \\
\hline
\end{tabular}

\subsection{Effect of Optimal DG Placement on IEEE-30 System Losses}

While the objective function is to enhance minimum voltage profile, the effect of optimal DGs on system losses were monitored. The results were tabulated in Table 4 and Table 5. Along with the system losses, the percentage of losses reduction (LRP) was calculated using (8). Based on this equation, if system loss with optimal DG insertion is less than system loss without DG, then LRP value will be positive number.

$$
L R P=\frac{\text { Loss }_{\text {pre-opt }}-\text { Loss }_{D G}}{\text { Loss }_{\text {pre }- \text { opt }}} \times 100 \%
$$

As the reactive load increases, optimal T1 and T3 DGs show their ability to consistently reduce the system loss while enhancing the minimum voltage profile. When healthy Bus-6 was burdened with increasing reactive load, T1 DGs shows better performance than T3 DGs in reducing the loss as indicated by high LRP values. Also, five T1 DGs outperform the two or three DGs units about 20 points. However, when weak Bus-30 reactive load increases, optimal T3 DGs are better than T1 DGs in many occasions and show better consistency in reducing total system loss as DG units increases. When $\mathrm{Q}_{\mathrm{d} 30}=30 \mathrm{MVar}$, five optimal T1 DGs were able to put the system into the healthy-mode but caused very high system loss. As for T2 and T4 DGs, IBSEP technique was not able to determine the optimal sizes and locations such that system's minimum voltage profile is improved and system loss is reduced indicated by a mix of positive and negative LRP values in Table 4 and Table 5. Therefore, if a utility provider is planning to improve its transmission system performance in terms of stable network while not negatively affected its total loss at any loading scenarios, then installing T3 types DGs would be a better decision. Table 6 lists all optimal DG locations and sizes determined by IBSEP technique to compensate for IEEE-30 RTS minimum voltage profile, when $\mathrm{Q}_{\mathrm{d} 30}$ were varied.

Table 4. IEEE-30 RTS System Losses and Loss Reduction Percentage (LRP) with Optimal DGs while $\mathrm{Q}_{6}$ increases

\begin{tabular}{cccccccccc}
\hline $\begin{array}{c}\mathrm{Q}_{\mathrm{d}_{6}} \\
(\mathrm{MVar})\end{array}$ & $\begin{array}{c}\text { DG Amt. } \\
\text { (unit) }\end{array}$ & $\begin{array}{c}\text { Loss }_{\mathrm{T} 1} \\
(\mathrm{MW})\end{array}$ & $\begin{array}{c}\text { Loss }_{\mathrm{T} 2} \\
(\mathrm{MW})\end{array}$ & $\begin{array}{c}\text { Loss }_{\mathrm{T} 3} \\
(\mathrm{MW})\end{array}$ & $\begin{array}{c}\text { Loss }_{\mathrm{T} 4} \\
(\mathrm{MW})\end{array}$ & $\begin{array}{c}\mathrm{LRP}_{\mathrm{T} 1} \\
(\%)\end{array}$ & $\begin{array}{c}\mathrm{LRP}_{\mathrm{T} 2} \\
(\%)\end{array}$ & $\begin{array}{c}\mathrm{LRP}_{\mathrm{T} 3} \\
(\%)\end{array}$ & $\begin{array}{c}\mathrm{LRP}_{\mathrm{T} 4} \\
(\%)\end{array}$ \\
\hline \multirow{3}{*}{0} & 2 & 10.21 & 17.68 & 13.53 & 14.61 & $\mathbf{4 1 . 9 6}$ & -0.48 & 23.11 & 17.00 \\
& 3 & 10.55 & 17.81 & 11.17 & 15277 & $\mathbf{4 0 . 0 5}$ & -1.23 & 36.52 & -86713 \\
& 5 & 6.25 & 19.96 & 10.87 & 30.58 & $\mathbf{6 4 . 5 0}$ & -13.39 & 38.26 & -73.79 \\
\multirow{3}{*}{10} & 2 & 10.23 & 17.68 & 13.53 & 16.51 & $\mathbf{4 2 . 2 3}$ & 0.17 & 23.61 & 6.79 \\
& 3 & 10.58 & 17.66 & 10.75 & 16.05 & $\mathbf{4 0 . 2 7}$ & 0.31 & 39.32 & 9.37 \\
& 5 & 6.84 & 19.93 & 10.86 & 13.13 & $\mathbf{6 1 . 3 9}$ & -12.50 & 38.71 & 25.90 \\
\multirow{2}{*}{20} & 2 & 10.27 & 17.70 & 13.55 & 15.34 & $\mathbf{4 2 . 2 0}$ & 0.36 & 23.73 & 13.62 \\
& 3 & 10.62 & 17.67 & 13.23 & 232.27 & $\mathbf{4 0 . 1 8}$ & 0.51 & 25.48 & -1208 \\
& 5 & 6.88 & 19.89 & 10.83 & 35.65 & $\mathbf{6 1 . 2 8}$ & -11.98 & 39.01 & -100.75 \\
& 2 & 10.31 & 17.72 & 11.85 & 23.66 & $\mathbf{4 2 . 1 4}$ & 0.54 & 33.51 & -32.78 \\
& 30 & 10.68 & 17.69 & 13.26 & 159.54 & $\mathbf{4 0 . 0 7}$ & 0.72 & 25.59 & -795.42 \\
& 5 & 6.92 & 19.95 & 10.97 & 73.18 & $\mathbf{6 1 . 1 3}$ & -11.99 & 38.43 & -310.71 \\
\hline
\end{tabular}


Table 5. IEEE-30 RTS System Losses and Loss Reduction Percentage (LRP) with Optimal DGs While $\mathrm{Q}_{\mathrm{d}_{30}}$

\begin{tabular}{cccccccccc}
\hline $\begin{array}{c}\mathrm{Q}_{\mathrm{d} 30} \\
(\mathrm{MVar})\end{array}$ & $\begin{array}{c}\text { DG Amt. } \\
\text { (unit) }\end{array}$ & $\begin{array}{c}\text { Loss }_{\mathrm{T} 1} \\
(\mathrm{MW})\end{array}$ & $\begin{array}{c}\text { Loss }_{\mathrm{T} 2} \\
(\mathrm{MW})\end{array}$ & $\begin{array}{c}\text { Loss }_{\mathrm{T} 3} \\
(\mathrm{MW})\end{array}$ & $\begin{array}{c}\text { Loss }_{\mathrm{T} 4} \\
(\mathrm{MW})\end{array}$ & $\begin{array}{c}\mathrm{LRP}_{\mathrm{T} 1} \\
(\%)\end{array}$ & $\begin{array}{c}\mathrm{LRP}_{\mathrm{T} 2} \\
(\%)\end{array}$ & $\begin{array}{c}\mathrm{LRP}_{\mathrm{T} 3} \\
(\%)\end{array}$ & $\begin{array}{c}\mathrm{LRP}_{\mathrm{T} 4} \\
(\%)\end{array}$ \\
\hline \multirow{3}{*}{0} & 2 & 12.12 & 17.67 & 11.80 & 16.84 & 30.97 & -0.59 & $\mathbf{3 2 . 8 1}$ & 4.13 \\
& 3 & 11.74 & 20.48 & 11.21 & 17.01 & 33.19 & -16.58 & $\mathbf{3 6 . 2 0}$ & 3.14 \\
& 5 & 8.60 & 19.94 & 10.85 & 520.79 & $\mathbf{5 1 . 0 2}$ & -13.51 & $\mathbf{3 8 . 2 5}$ & -2865 \\
& 2 & 11.41 & 18.89 & 12.11 & 91.05 & $\mathbf{3 6 . 9 7}$ & -4.32 & 33.15 & -402.79 \\
10 & 3 & 13.11 & 20.92 & 11.21 & 1568 & 27.61 & -15.54 & $\mathbf{3 8 . 1 0}$ & -8560 \\
& 5 & 9.64 & 19.43 & 9.96 & 1748.41 & $\mathbf{4 6 . 7 9}$ & -7.32 & 44.99 & -9555 \\
& 2 & 16.08 & 19.83 & 14.45 & 41.25 & 17.73 & -1.45 & $\mathbf{2 6 . 0 9}$ & -111.04 \\
& 3 & 14.57 & 20.66 & 11.26 & 157.56 & 25.44 & -5.71 & $\mathbf{4 2 . 3 8}$ & -706.02 \\
& 5 & 11.28 & 23.15 & 8.96 & 10744 & 42.30 & -18.40 & $\mathbf{5 4 . 1 5}$ & -54862 \\
& 2 & 18.80 & 20.07 & 15.06 & 14.40 & 19.81 & 14.37 & 35.76 & $\mathbf{3 8 . 5 7}$ \\
& 30 & 17.69 & 20.78 & 11.70 & 62.96 & 24.53 & 11.37 & $\mathbf{5 0 . 1 0}$ & -168.56 \\
& 3 & 1082 & 20.99 & 9.39 & 16.67 & -4515 & 10.46 & $\mathbf{5 9 . 9 4}$ & 28.90 \\
\hline
\end{tabular}

Table 6. Optimal DG Location And Size Determined by IBSEP Technique to Enhance Minimum Voltage Profile Of IEEE-30 RTS as $\mathrm{Q}_{\mathrm{d} 30}$ Increases

\begin{tabular}{|c|c|c|c|c|c|c|c|c|c|}
\hline $\begin{array}{c}\mathrm{Q}_{\mathrm{d} 30} \\
\text { (MVar) }\end{array}$ & $\begin{array}{c}\text { DG Amt. } \\
\text { (unit) }\end{array}$ & $\begin{array}{c}\mathrm{X}_{\mathrm{T} 1} \\
\text { (Bus) }\end{array}$ & $\begin{array}{c}\mathrm{X}_{\mathrm{T} 2} \\
\text { (Bus) } \\
\end{array}$ & $\begin{array}{c}\mathrm{X}_{\mathrm{T} 3} \\
\text { (Bus) } \\
\end{array}$ & $\begin{array}{c}\mathrm{X}_{\mathrm{T} 4} \\
\text { (Bus) }\end{array}$ & $\begin{array}{c}\mathrm{S}_{\mathrm{T} 1} \\
(\mathrm{MVA})\end{array}$ & $\begin{array}{c}\mathrm{S}_{\mathrm{T} 2} \\
(\mathrm{MVA})\end{array}$ & $\begin{array}{c}\mathrm{S}_{\mathrm{T} 3} \\
(\mathrm{MVA}) \\
\end{array}$ & $\begin{array}{c}\mathrm{S}_{\mathrm{T} 4} \\
(\mathrm{MVA})\end{array}$ \\
\hline \multirow{2}{*}{0} & 2 & 7,26 & 7,26 & 19,21 & 12,17 & $35.7,8.0$ & $35.7,8.0$ & $36.4,43.0$ & $7.8,1.6$ \\
\hline & 5 & $\begin{array}{c}19,25 \\
22,29 \\
9\end{array}$ & $\begin{array}{c}3,3, \\
12,28, \\
14\end{array}$ & $\begin{array}{c}3,3 \\
12,28 \\
14\end{array}$ & $\begin{array}{c}30,30, \\
30,21, \\
\quad 21\end{array}$ & $\begin{array}{c}22.0,34.5 \\
49.4,42.6 \\
26.9\end{array}$ & $\begin{array}{c}47.4,25.0, \\
13.9,26.5, \\
\quad 47.1\end{array}$ & $\begin{array}{c}46.9,24.8 \\
13.7,26.2 \\
46.6\end{array}$ & $\begin{array}{l}35.9,43.8 \\
49.2,42.6 \\
\quad 46.5\end{array}$ \\
\hline \multirow{3}{*}{10} & 2 & 15,30 & 22,25 & 22,25 & 30,30 & $43.1,22.2$ & $49.4,34.5$ & $48.9,34.1$ & $38.36,7.22$ \\
\hline & 3 & $\begin{array}{c}15,25 \\
30\end{array}$ & $\begin{array}{c}22,25 \\
29\end{array}$ & $\begin{array}{c}16,21 \\
29\end{array}$ & $\begin{array}{c}22,22 \\
30\end{array}$ & $\begin{array}{c}18.4,8.1 \\
\quad 43.2\end{array}$ & $\begin{array}{c}49.4,34.5 \\
42.6\end{array}$ & $\begin{array}{c}41.1,33.6 \\
19.0\end{array}$ & $\begin{array}{c}25.21, \\
16.35,44.45\end{array}$ \\
\hline & 5 & $\begin{array}{c}30,25 \\
21,19 \\
12\end{array}$ & $\begin{array}{c}22,27 \\
10,16 \\
26\end{array}$ & $\begin{array}{l}22,27 \\
10,16 \\
26\end{array}$ & $\begin{array}{c}10,28 \\
26,26 \\
17\end{array}$ & $\begin{array}{c}47.7,38.9 \\
42.1,38.4, \\
29.3\end{array}$ & $\begin{array}{l}50.0,11.7, \\
17.6,29.6, \\
20.6\end{array}$ & $\begin{array}{c}49.5,11.5 \\
17.4,29.3 \\
20.4\end{array}$ & $\begin{array}{c}0.5,4.8, \\
47.4,33.4 \\
22.3\end{array}$ \\
\hline \multirow{2}{*}{20} & 3 & $\begin{array}{c}15,25 \\
30\end{array}$ & $\begin{array}{c}22,25 \\
29\end{array}$ & $\begin{array}{c}22,25 \\
29\end{array}$ & $\begin{array}{c}27,29 \\
30\end{array}$ & $\begin{array}{l}18.4,8.1 \\
\quad 43.2\end{array}$ & $\begin{array}{l}49.4,42.6 \\
\quad 34.5\end{array}$ & $\begin{array}{c}48.9,34.1 \\
42.1\end{array}$ & $\begin{array}{c}45.6,30.4 \\
14.7\end{array}$ \\
\hline & 5 & $\begin{array}{c}30,25 \\
21,19 \\
12\end{array}$ & $\begin{array}{c}28,26 \\
16,25 \\
14\end{array}$ & $\begin{array}{c}19,25 \\
22,29 \\
9\end{array}$ & $\begin{array}{c}25,10 \\
29,6,25\end{array}$ & $\begin{array}{c}47.7,38.8, \\
42.1,38.4, \\
\quad 29.3\end{array}$ & $\begin{array}{c}12.5,23.6, \\
30.0,37.8, \\
\quad 47.6\end{array}$ & $\begin{array}{c}21.8,34.1 \\
48.9,42.1 \\
26.6\end{array}$ & $\begin{array}{l}27.9,27.4 \\
35.6,14.8, \\
\quad 7.6\end{array}$ \\
\hline \multirow{3}{*}{30} & 2 & 10,30 & 6,29 & 6,29 & 19,30 & $2.9,27.1$ & $18.6,48.2$ & $18.4,47.8$ & $0.3,26.1$ \\
\hline & 3 & $\begin{array}{c}15,25 \\
30\end{array}$ & $\begin{array}{c}22,25 \\
29\end{array}$ & $\begin{array}{c}22,25 \\
29\end{array}$ & $\begin{array}{c}20,26 \\
30\end{array}$ & $\begin{array}{l}18.4,8.1 \\
\quad 43.2\end{array}$ & $\begin{array}{c}49.4,34.5 \\
42.6\end{array}$ & $\begin{array}{c}48.9,34.1 \\
42.1\end{array}$ & $\begin{array}{c}32.5,9.5 \\
22.7\end{array}$ \\
\hline & 5 & $\begin{array}{c}4,30 \\
16,30 \\
30\end{array}$ & $\begin{array}{c}19,25 \\
22,29 \\
9\end{array}$ & $\begin{array}{c}19,25 \\
22,29 \\
9\end{array}$ & $\begin{array}{c}29,4 \\
17,9,4\end{array}$ & $\begin{array}{c}44.1,28.2 \\
4.3,42.2 \\
14.6\end{array}$ & $\begin{array}{c}22.0,34.5, \\
49.4,42.6, \\
26.9\end{array}$ & $\begin{array}{c}21.8,34.1 \\
48.9,42.1 \\
26.6\end{array}$ & $\begin{array}{c}43.5,36.8 \\
42.8,47.0 \\
5.7\end{array}$ \\
\hline
\end{tabular}

\section{CONCLUSION}

This paper has presented effect of multiple different optimal DG types in terms of power delivering capabilities towards transmission network minimum voltage profile while monitoring the system's total loss. It can be concluded that optimal DGs that provide reactive power could enhance the minimum voltage profile the most in many scenarios. Other DG types may compensate the voltage profile degradation in most cases, but may cause more power loss. IBSEP optimization technique was able to determine optimal sizes and location for all DG types and amount, in order to fulfill the objective function of increasing minimum voltage profile. 


\section{ACKNOWLEDGEMENTS}

The authors would like to acknowledge Universiti Tenaga Nasional, Selangor, Malaysia for the financial support of this research. This research is supported by Universiti Tenaga Nasional under the Universiti Tenaga Nasional Internal Grant (UNIIG2017) with project code: J510050685.

\section{REFERENCES}

[1] J. Vanishree and V. Ramesh, "Voltage profile improvement in power systems - A review," in 2014 International Conference on Advances in Electrical Engineering, ICAEE 2014, 2014.

[2] T. Ackermann, et al, "Distributed generation: a definition," Electr. Power Syst. Res., vol. 57, no. 3, pp. 195-204, Apr. 2001.

[3] G. Pepermans, et al, "Distributed generation : definition, benefits and issues," vol. 33, pp. 787-798, 2005.

[4] C. R. Sarimuthu, V. K. Ramachandaramurthy, H. Mokhlis, and K. R. Agileswari, "Impact of distributed generation on voltage profile in radial feeder," Indones. J. Electr. Eng. Comput. Sci., vol. 6, no. 3, pp. 583-590, Jun. 2017.

[5] B. Poornazaryan, et al, "Optimal allocation and sizing of DG units considering voltage stability, losses and load variations," Int. J. Electr. Power Energy Syst., vol. 79, pp. 42-52, Jul. 2016.

[6] U. Sultana, et al, "A review of optimum DG placement based on minimization of power losses and voltage stability enhancement of distribution system," Renew. Sustain. Energy Rev., vol. 63, pp. 363-378, 2016.

[7] S. A. S. Mustaffa, et al, "Multi DGPV installation in transmission system for loss minimization," in 20174 th International Conference on Industrial Engineering and Applications, ICIEA 2017, 2017, pp. 350-354.

[8] D. Q. Hung, et al, "Integration of PV and BES units in commercial distribution systems considering energy loss and voltage stability," Appl. Energy, vol. 113, pp. 1162-1170, Jan. 2014.

[9] E. Mahboubi-Moghaddam et al, "Multi-Objective Distribution feeder reconfiguration to improve transient stability, and minimize power loss and operation cost using an enhanced evolutionary algorithm at the presence of distributed generations," Int. J. Electr. Power Energy Syst., vol. 76, pp. 35-43, Mar. 2016.

[10] S. A. Shaaya, et al, "Performance comparison of distributed generation installation arrangement in transmission system for loss control," vol. 8, no. 1, pp. 39-45, 2019.

[11] S. A. Shaaya, et al, "Optimal DGPV Installation in Transmission System Using Immunized-BrainstormEvolutionary Programming for Loss Minimization,” J. Fundam. Appl. Sci., vol. 10, no. No 7S, pp. 183-198, 2018.

[12] S. A. S. Mustaffa, et al, "Chaotic mutation immune evolutionary programming for voltage security with the presence of DGPV," Indones. J. Electr. Eng. Comput. Sci., 2017.

[13] R. P. Payasi, et al, "Optimal Location and Size of Different Type of Distributed Generation with Voltage Step Constraint," pp. 341-357, 2013.

[14] M. F. Nadeem, et al, "Simultaneous placement and sizing of multi-DG units in distribution system using IGSA," in 2018 International Conference on Computing, Mathematics and Engineering Technologies: Invent, Innovate and Integrate for Socioeconomic Development, iCoMET 2018 - Proceedings, 2018.

[15] M. H. Mansor, et al, "Log-Normal Based Mutation Evolutionary Programming Technique for Solving Economic Dispatch,” Int. Multi-disciplinary Grad. Conf. Teren. 2016 (GRaCe 2016), no. GraCe, pp. 1-8, 2016.

[16] M. K. Mohamad Zamani, et al, "Active and reactive power scheduling optimization using firefly algorithm to improve voltage stability under load demand variation," Indones. J. Electr. Eng. Comput. Sci., vol. 9, no. 2, 2018.

[17] J. Soares, et al, "Distributed energy resource short-term scheduling using Signaled Particle Swarm Optimization," Energy, vol. 42, no. 1, pp. 466-476, 2012.

[18] Z. M. Yasin, et al, "Multiobjective Quantum-inspired Evolutionary Programming for Optimal Location and Sizing of Distributed Generation,” 2012 IEEE Conf. Sustain. Util. Dev. Eng. Technol. STUDENT 2012 - Conf. Bookl., pp. 233-238, 2012.

[19] G. Yang-yang, et al, "Optimal placement for hybrid energy in micro-grid," 2016 IEEE Int. Conf. Power Syst. Technol., vol. 3, pp. 1-8, 2016.

[20] D. R. Prabha, et al, "Optimal Location and Sizing of Distributed Generation Unit using Intelligent Water Drop Algorithm," Sustain. Energy Technol. Assessments, vol. 11, pp. 106-113, 2015.

[21] I. Rahman and J. Mohamad-saleh, "Hybrid bio-Inspired computational intelligence techniques for solving power system optimization problems : A comprehensive survey," Appl. Soft Comput. J., vol. 69, pp. 72-130, 2018.

[22] K.Lenin, "Shrinkage of real power loss by enriched brain storm optimization algorithm," IAES Int. J. Artif. Intell., vol. 8, no. 1, pp. 1-6, 2019.

[23] Z. Song, et al, "A Simple Brain Storm Optimization Algorithm with a Periodic Quantum Learning Strategy," IEEE Access, vol. 6, pp. 19968-19983, 2017.

[24] S. H. I. Cheng, et al, "Locating Multiple Optima via Brain Storm Optimization Algorithms," vol. 6, pp. 1703917049, 2018.

[25] A. Cervantes-Castillo and E. Mezura-Montes, "A study of constraint-handling techniques in brain storm optimization," in 2016 IEEE Congress on Evolutionary Computation, CEC 2016, 2016.

[26] Z. Yang and Y. Shi, "Brain Storm Optimization with Chaotic Operation," in 7th Int. Conference on Advanced Computational Intelligence, pp. 111-115.

[27] A. W. H. Sie et al, "A methodology to determine suitable placement of solar photovoltaic sources in the transmission system taking into account Voltage Stability Index (VSI)," in Conference Proceeding - 2014 IEEE International Conference on Power and Energy, PECon 2014, 2014, pp. 226-230. 


\section{BIOGRAPHIES OF AUTHORS}
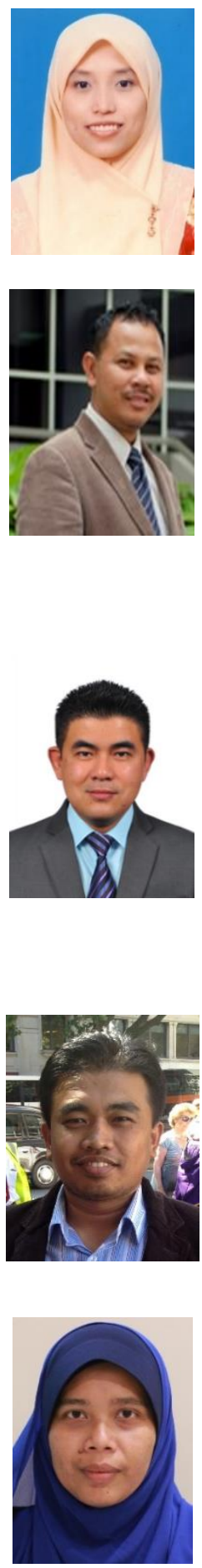

Sharifah Azwa Shaaya obtained her Master of Engineering in Electrical-electronics and Telecommunications from Universiti Teknologi Malaysia (UTM) in 2005 and her Bachelor of Electrical and Electronics Engineering from Universiti Tenaga Nasional (UNITEN), Malaysia in 2001. She is currently a lecturer at the College of Engineering, UNITEN while pursuing her $\mathrm{PhD}$ in Universiti Teknologi Mara, (UiTM), in Shah Alam, Malaysia. Her research interests focus on the application of computational intelligence in solving power system problems, mostly on distributed generation and power scheduling.

Prof. Dr. Ismail Musirin obtained Bachelor of Electrical Engineering (Hons) in 1990 from Universiti Teknologi Malaysia, MSc Pulsed Power Technology in 1992 from University of Strathclyde, United Kingdom and $\mathrm{PhD}$ in Electrical Engineering from Universiti Teknologi MARA (UiTM), Malaysia in 2005. He is currently a Professor at the Faculty of Electrical Engineering, UiTM. He also leads the Power System Operation Computational Intelligence Research Group (POSC) at UiTM. He has authored and co-authored 2 books, over 300 technical papers in indexed international journal and conferences. He is a Senior Member of International Association of Computer Science and Information Technology (IACSIT), member of Artificial Immune System Society (ARTIST), member of International Association of Engineers (IAENG) and member for Association of Energy Engineers (AEE). To date he has conducted numerous short courses for local universities in Malaysia and industries.

Shahril Irwan Sulaiman holds a $\mathrm{PhD}$ in Electrical Engineering from Universiti Teknologi MARA, Malaysia. He obtained his M.EngSc in Photovoltaic Engineering from University of New South Wales, Australia and B.Eng in Electrical \& Electronics from Universiti Tenaga Nasional, Malaysia. He is an associate professor in Faculty of Electrical Engineering, Universiti Teknologi MARA, Malaysia and the Head of Green Energy Research Centre. Besides conducting numerous contract-based research, he has been assisting the national photovoltaic industry and the government for more than a decade. He regularly conducts competency-based trainings related to design, installation, testing \& commissioning, operation and maintenance of both grid-connected photovoltaic systems and stand-alone photovoltaic systems. He is also a member of standards working group on photovoltaic systems and has been prominently involved in preparing various policies and guidelines related to photovoltaic systems in Malaysia.

Mohd Helmi Mansor was born in Johor, Malaysia, on September 18, 1983. Mohd Helmi Mansor received his Bachelor of Engineering (Hons) Electrical Power in 2008 from Universiti Teknologi MARA (UiTM) and MSc Sustainable Electrical Power in 2009 from Brunel University, United Kingdom. He was an electrical engineer at TNB planning division in 2008 before joining Universiti Tenaga Nasional (UNITEN) as a tutor at College of Engineering. He is currently a lecturer in UNITEN teaching bachelor of electrical power engineering students. His research interests focus on the application of computational intelligence in solving power system problems such as economic dispatch of generation, FACTS devices installation, state estimation, network planning and distributed generation.

Sharifah Azma Syed Mustaffa received her B.Eng Electrical Power (Hons) in 2002 from Universiti Tenaga Nasional (UNITEN), Malaysia and M.Eng. Electrical Engineering (Power) from Universiti Teknologi Malaysia (UTM), Malaysia in 2006. She is currently pursuing her Ph.D. degree with the Faculty of Electrical Engineering, Universiti Teknologi MARA (UiTM), Malaysia. Her research interest includes voltage stability, and optimization techniques in power system application. 